\title{
Evidence for particle acceleration in the magnetospheric cusp
}

\author{
J. Chen \\ Dept. of Astronomy \& Center for Space Physics, Boston University, 725 Commonwealth Avenue, Boston, MA 02215, USA
}

Received: 1 May 2008 - Revised: 17 June 2008 - Accepted: 30 June 2008 - Published: 23 July 2008

\begin{abstract}
New evidence reveals that the charged particles can be energized locally in the magnetospheric cusp. The power spectral density of the cusp magnetic fluctuations shows increases by up to four orders of magnitude in comparison to an adjacent region. Large fluctuations of the cusp electric fields have been observed with an amplitude of up to $350 \mathrm{mV} / \mathrm{m}$. The measured left-hand polarization of the cusp electric field at ion gyro-frequencies indicates that the cyclotron resonant acceleration mechanism is working in this region. The cyclotron resonant acceleration can energize ions from $\mathrm{keV}$ to $\mathrm{MeV}$ in seconds.
\end{abstract}

Keywords. Magnetospheric physics (Electric fields; Magnetopause, cusp, and boundary layers) - Space plasma physics (Charged particle motion and acceleration)

\section{Introduction}

For decades, the Earth's magnetospheric cusp has been considered as only a sink - a very narrow and small region of weak magnetic fields with funnel-shaped field lines that map to the dayside and nightside of the magnetopause surface (Chapman and Ferraro, 1931; Roederer, 1970). No significant energetic particle fluxes were expected to be detected there (Roederer, 1970). Therefore, it came with a big surprise when the POLAR spacecraft measured temporarily confined $\mathrm{MeV}$ charged particles in the high-altitude cusp region (Chen et al., 1997, 1998; Sheldon et al., 1998). Recent multiple satellite (POLAR and CLUSTER) measurements reveal that the Earth's magnetospheric cusp is a broad and dynamic region. It has a size of several $R_{E}$ (Earth radii) (Chen and Fritz, 2002; Chen et al., 2005a). Associated with this region are charged particles with energies from $20 \mathrm{keV}$ up to $15 \mathrm{MeV}$ that are potentially an additional independent source of the

Correspondence to: J. Chen

(jschen@bu.edu) charged particles in the outer Van Allen radiation belt (Chen et al., 2005b). However, how these cusp energetic particles are energized remains unknown. The present study reports new observations of large amplitude electromagnetic fluctuations in the magnetospheric cusp that can effectively accelerate cusp charged particles.

\section{Association of cusp energetic particles with diamag- netic cavities}

The POLAR spacecraft often measured MeV charged particles with depressed magnetic field in the high-altitude cusp region (Chen et al., 1997, 1998; Sheldon et al., 1998). One example is shown in Fig. 1. Figure 1a and b compares the $\mathrm{MeV}$ helium flux with the local magnetic field measured by POLAR on 10 September 1996. It shows two cusp locations with the first one around 10.5 MLT (magnetic local time) at 04:08-04:36 UT and the second one between 13.114.5 MLT at 06:12-09:30 UT. At the first location, the geomagnetic field strength changed from $120 \mathrm{nT}$ to $2 \mathrm{nT}$. Generally, the gyro-motions of the charged particles in an imposed magnetic field can induce a magnetic field opposed to the imposed field; the resulting magnetic field is depressed and is called the diamagnetic cavity. An association (anticorrelation) of the cusp energetic particle (CEP) intensities with the diamagnetic cavities is obvious from Fig. 1, which is an observational feature of the CEP events.

This feature has also been confirmed by the CLUSTER mission. The CLUSTER mission consists of four identical satellites (C1, C2, C3, and C4). On 5 March 2001, while crossing through the dayside high-altitude cusp region in the Northern Hemisphere, the CLUSTER detected simultaneous enhancements of the energetic electron and ion fluxes. Figure 2 shows the measurements of the $>100 \mathrm{keV}$ electrons, 30-100 keV electrons, 30-100 keV protons and $50-150 \mathrm{keV}$ helium ions (top three panels) with the local

Published by Copernicus Publications on behalf of the European Geosciences Union. 


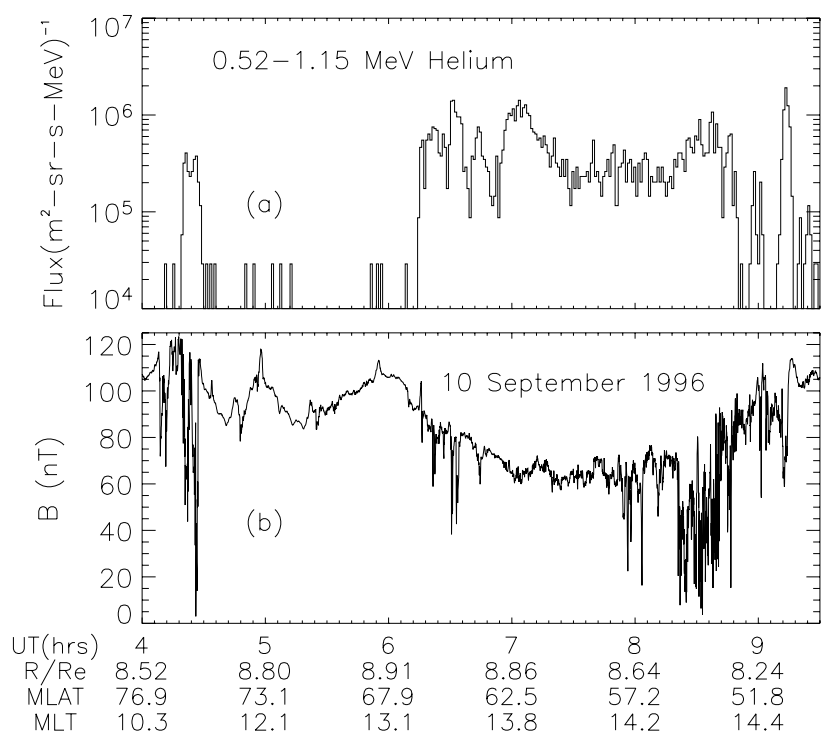

Fig. 1. POLAR measurement for the $0.52-1.15 \mathrm{MeV}$ Helium flux versus time (a) at 04:00-09:30 UT on 10 September 1996, and the corresponding variations of the geomagnetic field (b), followed by the distance of POLAR from the Earth (in $R_{E}$ ), the magnetic latitude (MLAT), and the magnetic local time (MLT).

magnetic field strength (bottom panel) by the $\mathrm{C} 4$ satellite over 07:30-11:00 UT on 5 March 2001. It shows the increased intensities of the charged particles was associated with the large diamagnetic cavities. Since the $30-100 \mathrm{keV}$ and $>100 \mathrm{keV}$ relativistic electrons move at $32-55 \%$ and $>55 \%$ of the speed of light, respectively, and since inside the geomagnetic field the electron and the proton drift in opposite directions, it is unexpected to detect them simultaneously in the cusp diamagnetic cavities for hours. Note that at $08: 50-10: 00$ UT the $30-100 \mathrm{keV}$ proton flux in the cusp was about three to four orders of magnitude higher than that before 07:40 UT and after 10:45 UT when CLUSTER was outside the cusp region. The top two panels show no obvious energy dispersion signatures for the relativistic electrons, suggesting a local dynamic process.

\section{Large electromagnetic fluctuations found in the cusp diamagnetic cavities}

An indicator of the dynamic processes is magnetic fluctuations that are associated with the charged particles (Blecki et al., 1999). As shown in Fig. 1, strong electromagnetic fluctuations are found in the large cusp diamagnetic cavities. A difference method of spectral analysis (Chen, 1989; Bieber et al., 1993) was used to determine the power spectra of the magnetic field for fluctuations in the ultra low frequency (ULF) range of 0.02 to $3 \mathrm{~Hz}$ over three 60 -s periods on 10 September 1996. The results were plotted in the left panel of Fig. 3. POLAR was inside the cusp diamagnetic cavities at

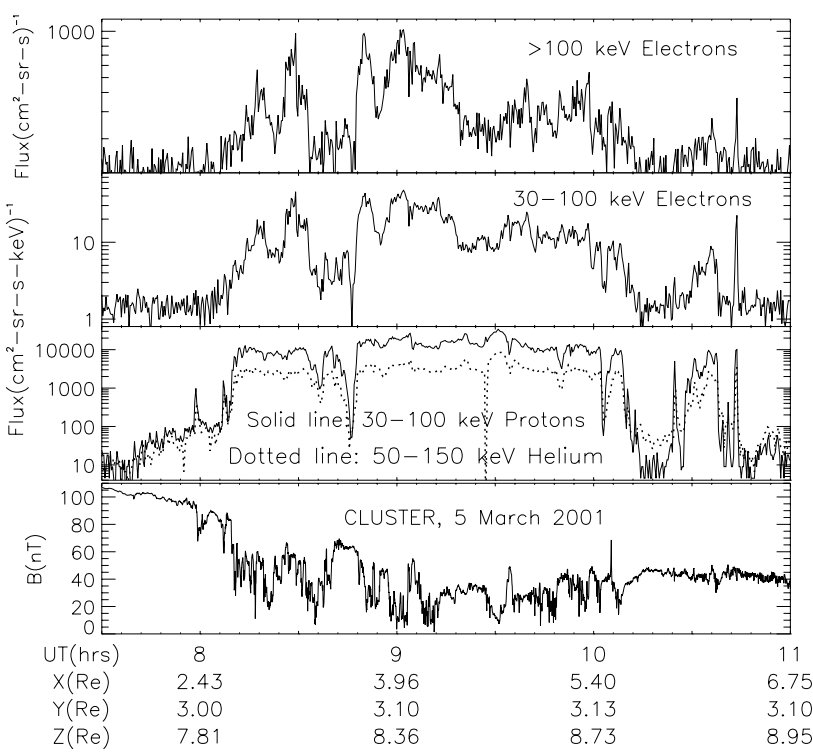

Fig. 2. Time variations of the cusp energetic particles and magnetic field strength. Panels from top to bottom are the time-intensity profiles of the $>100 \mathrm{keV}$ electrons, the $30-100 \mathrm{keV}$ electrons, the $30-100 \mathrm{keV}$ protons and $50-150 \mathrm{keV}$ helium ions, and the magnetic field strength measured by the CLUSTER satellite (C4) on 5 March 2001. The distance of CLUSTER from the Earth (in $R_{E}$ ) is shown at the bottom in the Geocentric Solar Ecliptic coordinates.

08:36-08:37 UT and 07:49-07:50 UT, and outside the cavity at 06:06-06:08 UT. When compared to 06:06-06:08 UT, the ULF power spectral density of the cavity magnetic field at 08:36-08:37 UT increases by more than four orders of magnitude. The power spectra densities also exhibit notable enhancements around the $\mathrm{He}^{++}$and proton gyro-frequencies depicted by arrows. The right panel of Fig. 3 displays the perpendicular and parallel power spectra of the cusp magnetic field for fluctuations in the ULF range at 07:24-07:25 UT. It shows two obvious features: (i) the spectral density perpendicular to the magnetic field dominates over the parallel one, and (ii) two spectral peaks occurred at the $\mathrm{He}^{++}$and proton gyro-frequencies of $\sim 0.48 \mathrm{~Hz}$ and $0.95 \mathrm{~Hz}$. The 60 -s interval covers $\sim 29$ and 57 gyro-periods for $\mathrm{He}^{++}$and proton, respectively. Over the ULF range, the power spectral density in the cusp dominated by the perpendicular component is also true for other time intervals including the two cases shown in the left panel of Fig. 3 at 07:49-07:50 UT and 08:3608:37 UT. It is a permanent feature that the magnetic power density in the cusp diamagnetic cavities exhibits orders of magnitude enhancement when compared to the adjacent regions. Such magnetic power enhancements in the cusp diamagnetic cavities have also been detected at higher frequencies from $6 \mathrm{~Hz}$ to several $\mathrm{kHz}$ (the electron gyro-frequency) (Chen et al., 1998; Pickett et al., 1999).

Figure 4 is the cusp electric field (top three panels) and magnetic field strength (bottom panel) measured by the 

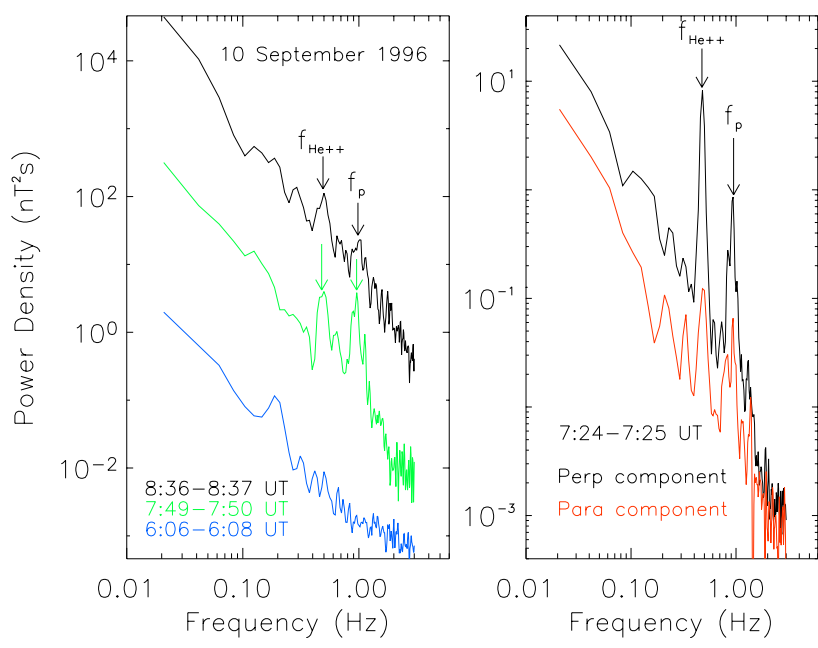

Fig. 3. The left panel is the total magnetic power spectra for fluctuations in the ULF range at three different 60 -s periods on 10 September 1996. The right panel is the perpendicular and parallel power spectra at 07:24-07:25 UT on that day.

POLAR satellite on 28 June 1999 when POLAR was at position of $\sim 7.7 R_{E}, 27^{\circ}$ MLAT (magnetic latitude), and 07:00 MLT, where the $E_{x}$ and $E_{y}$ are two electric field components perpendicular to the magnetic field and the $E$ is the total electric field. It displays large electromagnetic fluctuations. The cusp magnetic field strength varied between $260 \mathrm{nT}$ and $0 \mathrm{nT}$, which will generate an inductive electric field. The top two panels of Fig. 4 show that the $E_{x}$ changed between $-200 \mathrm{mV} / \mathrm{m}$ and $240 \mathrm{mV} / \mathrm{m}$, while $E_{y}$ varied between $-300 \mathrm{mV} / \mathrm{m}$ and $350 \mathrm{mV} / \mathrm{m}$. Since the gyro-frequency of a charged particle is proportional to the magnetic field strength, some of the cusp charged particles have to undergo a resonant or partial resonant acceleration with the varying electric field. At the cusp diamagnetic cavity edge (around 05:13 UT) the electric field had a value of $\sim 150 \mathrm{mV} / \mathrm{m}$. Inside the diamagnetic cavities, the measured cusp electric field peaked at $350 \mathrm{mV} / \mathrm{m}$. Compared to the reported equatorial convection electric field of less than $0.16 \mathrm{mV} / \mathrm{m}$ (Kosik, 1979), the measured cusp electric field of $350 \mathrm{mV} / \mathrm{m}$ is much higher by more than three orders of magnitude, and its power (proportional to square of the electric field) is much larger by more than six orders of magnitude. Figures 3 and 4 reveal that the cusp diamagnetic cavities are an extraordinarily dynamic region. The variations of the cusp electric field must change the charged particle energy with some of them being energized locally.

\section{Resonant acceleration in the cusp diamagnetic cavi- ties}

The magnetic power spectra with peaks at the ion gyrofrequencies shown in Fig. 3 suggest a cyclotron resonant

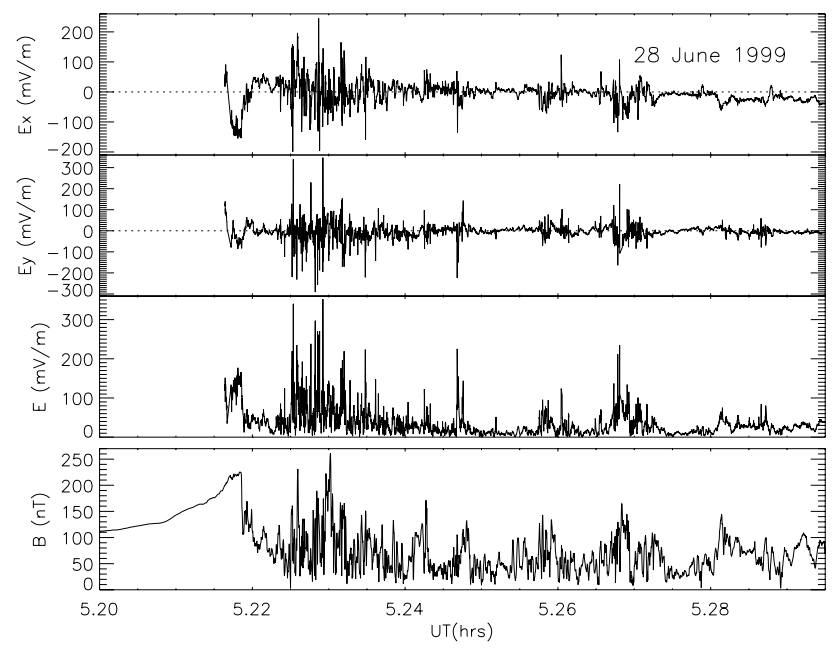

Fig. 4. The time variations of the cusp electric field (Top three panels), and the local magnetic field strength (bottom) measured by POLAR at 05:12:00-05:17:42 UT on 28 June 1999.
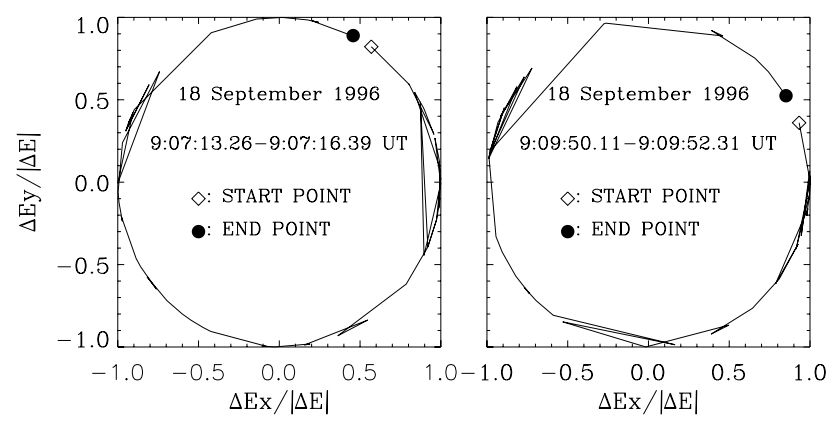

Fig. 5. Hodograms of the two perpendicular components of the cavity electric field at two different time periods on 18 September 1996, where the $|\Delta E|=|E-<E>|, \Delta E_{x}=E_{x}-<E_{x}>$, and $\Delta E_{y}=E_{y}-<E_{y}>$ with $<>$ being the average over the time period.

interaction of the field with the charged particles. On 18 September 1996, during the period of 07:00-10:06 UT, when POLAR was at $8.3-8.9 R_{E}, 56-70^{\circ}$ MLAT, and 13.4-14.3 MLT, it detected MeV charged particles and large diamagnetic cavities (Chen et al., 1998). Figure 5 is a hodogram of the two perpendicular components of the cusp electric field within this event period. In Fig. 5, the Z-axis (out of the page) points along the local magnetic field direction and $X$, Y-axes complete the right-handed system. In each panel, the time period, the start point and the end point are shown. This hodogram shows a left-hand circular polarization of the cusp electric field in the mean magnetic field-aligned coordinates over a period near the $\mathrm{He}^{+}$gyro-period (left panel) and the $\mathrm{O}^{+6}$ gyro-period (right panel). It can energize the helium and oxygen ions by a cyclotron resonant acceleration mechanism. Figure 5 demonstrates that the cyclotron resonant acceleration works in the cusp diamagnetic cavities. 
The energization rate by the cyclotron resonant acceleration can be expressed analytically by the measurable terms. By definition, the changing rate of the kinetic energy of a charged particle with velocity $\boldsymbol{v}$ in electric field $\boldsymbol{E}$ and magnetic field $\boldsymbol{B}$ is

$d K / d t=\boldsymbol{F} \cdot \boldsymbol{v}$,

where $\boldsymbol{F}=q(\boldsymbol{E}+\boldsymbol{v} \times \boldsymbol{B})$ is the Lorentz force with $q$ being the particle charge. Since $(\boldsymbol{v} \times \boldsymbol{B}) \cdot \boldsymbol{v}=0$ (the magnetic force is always perpendicular to the $\boldsymbol{v}$ and does no work on the particle), Eq. (1) is equivalent to

$d K / d t=q \boldsymbol{E} \cdot \boldsymbol{v}$.

Equation (2) contains both perpendicular and parallel components. For particle cyclotron resonant acceleration, one only needs to consider the perpendicular component; that is,

$d K_{\perp} / d t=q E v_{\perp}$,

where $E$ is the left-hand polarization perpendicular electric field with frequency the same as the ion gyro-frequency, and $v_{\perp}$ is the particle's perpendicular velocity. For nonrelativistic ions, $v_{\perp}=\left(2 K_{\perp} / m\right)^{1 / 2}$, so that Eq. (3) becomes

$d K_{\perp} / d t=\left(2 K_{\perp} / m\right)^{1 / 2} q E$,

or

$d K_{\perp} /\left(K_{\perp}\right)^{1 / 2}=(2 / m)^{1 / 2} q E d t$,

where $m$ is the ion mass. Integration of Eq. (5) gives the solution

$\left(K_{\perp}\right)^{1 / 2}=\left(K_{\perp}(0)\right)^{1 / 2}+(2 m)^{-1 / 2} q E t$,

where the $K_{\perp}(0)$ is the ion perpendicular kinetic energy at time $(t)=0$. Substituting Eq. (6) into Eq. (4) yields the changing rate of the ion perpendicular kinetic energy by the ion cyclotron resonant acceleration:

$d K_{\perp} / d t=\left(2 K_{\perp}(0) / m\right)^{1 / 2} q E+q^{2} E^{2} t / m$.

Over an ion gyro-period $\left(T_{i}\right)$, the energy increased due to the ion cyclotron resonant acceleration is

$\Delta K_{\perp}=\left(2 K_{\perp}(0) / m\right)^{1 / 2} q E T_{i}+q^{2} E^{2} T_{i}^{2} /(2 m)$.

Equation (8) can be obtained by either squaring Eq. (6) or integrating Eq. (7).

The significance of Eq. (8) is that all terms on its right side are measurable and are independent of models and simulations. Equation (8) indicates that an ion energy enhanced by the gyro-resonant acceleration is a function of the initial perpendicular kinetic energy, charge/mass ratio, gyro-period of the ion and the left-hand polarization perpendicular electric field. Taking $\mathrm{He}^{++}$ion as an example. Assuming its initial perpendicular kinetic energy $=2 \mathrm{keV}$ (the solar wind as seed population), the enhanced energy by the gyro-resonant acceleration for the $\mathrm{He}^{++}$ion will be $101.4 \mathrm{keV}, 0.54 \mathrm{MeV}$, and $2.04 \mathrm{MeV}$ over a two-second gyro-period for the perpendicular electric field of $20 \mathrm{mV} / \mathrm{m}, 50 \mathrm{mV} / \mathrm{m}$, and $100 \mathrm{mV} / \mathrm{m}$, respectively.

\section{Conclusions}

In summary, recent satellite observations from POLAR and CLUSTER reveal a broad and dynamic region centered at the Earth's magnetospheric cusp. This region is filled with the energetic charged particles, large diamagnetic cavities, and strong electromagnetic fluctuations. Inside the cavities, both the energetic particle intensity and the power spectral density of the magnetic fluctuations show increases by up to four orders of magnitude in comparison to an adjacent region. Peak power spectral densities at the ion gyro-frequencies have been measured. Over the ULF range, the power spectral densities are dominated by the perpendicular component of the local magnetic field. The cavity electric fields have an amplitude of up to $350 \mathrm{mV} / \mathrm{m}$, and its perpendicular component varies from $-300 \mathrm{mV} / \mathrm{m}$ to $350 \mathrm{mV} / \mathrm{m}$. The measured left-hand polarization of the cusp electric field at ion gyrofrequencies demonstrates that the cyclotron resonant acceleration occurs in the diamagnetic cavities. The cyclotron resonant acceleration can energize ions from $\mathrm{keV}$ to $\mathrm{MeV}$ in seconds.

Acknowledgements. I thank F. S. Mozer for providing the POLAR electric field data and C. T. Russell for the magnetic field data. I am grateful to the NASA's open data policy that makes the spacecraft data accessible for the present study.

Topical Editor I. A. Daglis thanks one anonymous referee for her/his help in evaluating this paper.

\section{References}

Bieber, J. W., Chen, J., Matthaeus, W. H., Smith, C. W., and Pomerantz, M. A.: Long-term variations of interplanetary magnetic field spectra with implications for cosmic-ray modulation, J. Geophys. Res., 98, 3585-3603, 1993.

Blecki, J., Kossacki, K., Wronowski, R., et al.: Low-frequency plasma waves observed in the outer polar cusp, Adv. Space Res., 23(10), 1765-1768, 1999.

Chapman, S. and Ferraro, V. C. A.: A new theory of magnetic storms, J. Geophys. Res., 36, 171-186, 1931.

Chen, J.: Long-term modulation of cosmic rays in interplanetary magnetic turbulence, Ph.D. thesis, Univ. of Del., Delaware, 1989.

Chen, J., Fritz, T. A., Sheldon, R. B., et al.: A new, temporarily confined population in the polar cap during the August 27, 1996 geomagnetic field distortion period, Geophys. Res. Lett., 24, 14471450, 1997.

Chen, J., Fritz, T. A., Sheldon, R. B., et al.: Cusp energetic particle events: Implications for a major acceleration region of the magnetosphere, J. Geophys. Res., 103, 69-78, 1998.

Chen, J. and Fritz, T. A.: The global significance of the CEP events, in: Solar-Terrestrial Magnetic Activity and Space Environment, edited by: Wang, H. N. and Xu, R. L., COSPAR Colloquia Series, 14, 239-249, 2002.

Chen, J., Fritz, T. A., and Sheldon, R. B.: Multiple spacecraft observations of energetic ions during a high solar wind pressure event, J. Geophys. Res., 110, A11212, doi:10.1029/2005JA011043, 2005a. 
Chen, J., Fritz, T. A., and Sheldon, R. B.: Comparison of energetic ions in cusp and outer radiation belt, J. Geophys. Res., 110, A12219, doi:10.1029/2004JA010718, 2005b.

Kosik, J. C.: Quantitative Modeling of Magnetospheric Processes, edited by: Olson, W. P., Geophys. Monogr. Series, 21, 569-581, 1979.

Pickett, J. S., Gurnett, D. A., Menietti, J. D., et al.: Plasma waves observed during cusp energetic particle events and their Correlation with POLAR and AKEBONO satellite and ground data, Adv. Space Res., 24, 23-33, 1999.
Roederer, J. G.: Dynamics of Geomagnetically Trapped Radiation, Springer, New York, 1970.

Sheldon, R. B., Spence, H. E., Sullivan, J. S., Fritz, T. A., and Chen, J.: The discovery of trapped energetic electrons in the outer cusp, Geophys. Res. Lett., 25, 1825-1828, 1998. 